\title{
Lactic acid as an adjuvant marker in pregnancy-associated sepsis
}

\author{
R Agarwal, ${ }^{1} \mathrm{MS}(\mathrm{O} \& \mathrm{G}) ; \mathbf{R}$ K Yadav, ${ }^{1} \mathrm{MBBS}$; S Garg, ${ }^{1} \mathrm{MD}$ (Biochem); H Srivastava, ${ }^{1} \mathrm{MS}(\mathrm{O} \& \mathrm{G}) ;$ G Radhakrishnan, ${ }^{2} \mathrm{MS}(\mathrm{O} \& \mathrm{G})$; \\ A Tiwari, ${ }^{1} \mathrm{MS}(\mathrm{O} \& \mathrm{G})$
}

${ }^{1}$ Department of Obstetrics \& Gynaecology, University College of Medical Sciences \& Guru Teg Bahadur, Delhi, India

${ }^{2}$ Department of Obstetrics \& Gynaecology, University College of Medical Sciences \& Guru Teg Bahadur, Delhi, India

Corresponding author: R Agarwal (rachna_anila@yahoo.co.in)

\begin{abstract}
Background. Lactic acid level is one index which is currently actively researched for its role in pregnancy-associated sepsis (PAS). We aimed to quantify the severity of PAS using lactic acid levels.

Methods. All pregnant, post-abortal (2 weeks) and postpartum women (up to 6 weeks) with clinical sepsis were enrolled as per systemic inflammatory response syndrome criteria, and lactic acid levels were estimated at admission. Severe PAS was defined as one or more organ dysfunctions due to sepsis. The severity of obstetric sepsis was graded according to number of organ failures.

Results. There were 42 patients with non-severe PAS, and 58 with severe PAS. The $\geq 4 \mathrm{mmol} / \mathrm{L}$ lactic acid threshold had $88.10 \%$ specificity and $37.93 \%$ sensitivity for detection of severe PAS. A significant difference $(p=0.006)$ was found between lactic acid levels (mean (standard deviation (SD)) in cases with single $\mathrm{OF}(n=24 ; 3.02(0.92) \mathrm{mmol} / \mathrm{L}) \mathrm{v}$. multi-organ failure $(n=34 ; 3.70(0.81) \mathrm{mmol} / \mathrm{L})$. The mean $(\mathrm{SD})$ lactic acid levels $(3.76(0.97) \mathrm{mmol} / \mathrm{L})$ with any type of culture-positive PAS cases $(n=35)$ were significantly higher than in culture-negative PAS cases (2.95 (0.98); $p=0.0001)$.

Conclusions. Lactic acid level $\geq 4 \mathrm{mmol} / \mathrm{L}$ had reasonable specificity for severity and culture positivity in PAS. A serum lactic acid level $\geq 3 \mathrm{mmol} / \mathrm{L}$ was associated with more number of organ failures.
\end{abstract}

S Afr J Obstet Gynaecol 2018;24(1):8-10. DOI:10.7196/SAJOG.2018.v24i1.1219

Lactic acid is one of the products of anaerobic metabolism related to hypovolemia and hypoxia. Following the recognition of lactic acid importance in critical care management of sepsis in general, its use was extended to pregnancy-associated sepsis (PAS) ${ }^{[1]}$ The existing evidence on the subject is still scanty. ${ }^{[2-5]}$ The only focused study of lactic acid measurements in obstetric sepsis indicated it as a marker of severe infection, associated with positive blood cultures, increased risk of intensive care unit ICU and telemetry unit admission and longer hospital stays. ${ }^{[2]}$ Presently, the lactic acid estimations have been included in the diagnostic criteria of the Sepsis Obstetrics Score (SOS) and Surviving Sepsis Campaign care bundles. ${ }^{[3,6]}$

Low-income countries have high burdens of morbidity and mortality related to PAS. The increasing severity of PAS worsens the maternal prognosis, and therefore there is a strong need to develop parameters for its identification to optimise outcomes. Lactic acid level is one index that is currently being researched for its role in PAS. We therefore conducted this study with the aim of quantifying the severity of PAS using lactic acid levels. We also compared the culture positivity in obstetrics sepsis with lactic acid levels, to try to find a possible association between them.

\section{Methods}

Ethics clearance was obtained from the Institutional Ethical Committee and prior consent was obtained from all patients. All pregnant, post-abortal (2 weeks) and postpartum women (up to 6 weeks) with clinical sepsis were investigated using systemic inflammatory response syndrome criteria: mean arterial blood pressure $<65 \mathrm{mmHg}$; systolic blood pressure $\leq 90 \mathrm{mmHg}$; heart rate $\geq 110 / \mathrm{min}$; respiratory rate $\geq 22 / \mathrm{min}$; temperature $\geq 38^{\circ} \mathrm{C}$ or $\leq 36^{\circ} \mathrm{C}$; and leukocyte count $\geq 14000 / \mu \mathrm{L}$ or $<4000 / \mu \mathrm{L}$. ${ }^{[3]}$ Patients meeting $\geq 2$ of these criteria were classified as having PAS and enrolled as cases for our study.

Detailed clinical, pertinent laboratory and imaging tests were performed for all enrolled patients. Blood, high vaginal swab and pus (if present) swabs were sent for bacterial cultures and sensitivity. Specimens were considered culture-positive if single microbial growth was isolated in any of the above samples. Venous lactic acid levels were obtained, along with other haematological investigations, at admission. For organ failure assessment, the key body systems were assessed and monitored: respiratory, cardiac, kidney and liver. The important criteria representing organ failure used were: altered mental status; arterial hypoxaemia (the ratio of partial pressure of arterial oxygen and the fraction of inspired oxygen $\left(\mathrm{PaO}_{2} /\right.$ $\mathrm{FiO}_{2}$ ) $<300$ ); acute oliguria (urine output $<0.5 \mathrm{~mL} / \mathrm{kg} / \mathrm{h}$ for at least 2 hours); creatinine increase $>0.5 \mathrm{mg} / \mathrm{dL}$; coagulation abnormalities (INR $>1.5$ or a PTT $>60$ s); thrombocytopenia (platelet count $<100 \quad 000 / \mathrm{mL}$ ); hyperbilirubinaemia (plasma total bilirubin $>4 \mathrm{mg} / \mathrm{dL}$ ). ${ }^{[7]}$ Severe sepsis was defined as an infectionrelated to $\geq 1$ organ dysfunction within 24 hours of admission. ${ }^{[7]}$ Patients were further managed as per the hospital protocol and the patient's condition.

Lactic acid estimations were associated with severity of sepsis and culture positivity using parametric and non-parametric statistical methods (SPSS software version 20 (IBM Corp., USA)).

\section{Results}

There were 42 patients with non-severe PAS and 58 with severe PAS. The common causes of PAS were urinary tract infection (80\%), followed by chorioamnionitis (10\%), endometritis (5\%) and peritonitis, along with pyoperitoneum (5\%). The two groups were 
similar in age, BMI and socioeconomic characteristics. The mean (standard deviation (SD)) lactic acid level in our cohort was 3.23 (0.98) $\mathrm{mmol} / \mathrm{L}$ (range $1.2-5.4 \mathrm{mmol} / \mathrm{L}$ ). The statistical comparison between mean lactic acid levels in non-severe (2.97 (1.02) mmol/L; range 1.2 - $5.4 \mathrm{mmol} / \mathrm{L})$ v. severe PAS $(3.42(0.91) \mathrm{mmol} / \mathrm{L}$; range 1.3 - $4.6 \mathrm{mmol} / \mathrm{L})$ was not significant $(p=0.197)$. When a lactic acid level of $4 \mathrm{mmol} / \mathrm{L}$ was considered as a threshold, a statistically significant difference $(p=0.000)$ was found between non-severe and severe PAS (Table 1). The $\geq 4 \mathrm{mmol} / \mathrm{L}$ threshold had a positive predictive value of $81.48 \%$ (specificity $88.10 \%$ and sensitivity $37.93 \%$ ) for PAS severity. The best lactic acid diagnostics were seen at cutoff levels of $3.2 \mathrm{mmol} / \mathrm{L}$ (specificity 50\%; sensitivity $71 \%$ ). The statistical analysis established the high specificity (nearly 90\%) of lactic acid levels $\geq 4 \mathrm{mmol} / \mathrm{L}$ for severe sepsis prediction. Moreover, there was a steady increase in lactic acid level at admission with the highest number of organ failures in subjects with severe sepsis. The mean (SD) lactic acid (mmol/L) was $3.02(0.92)$ in a single organ failure (OF) $(n=24)$, and it increased to $4.01(0.43)$ in those with $\geq 4$ OF $(n=12)$. The mean values for 2 and 3 OFs ( $n=11$ each) were 3.25 $(0.92)$ and $3.89(0.80) \mathrm{mmol} / \mathrm{L}$, respectively. A significant difference $(p=0.006)$ was found in lactic acid levels for single $(3.02(0.92) \mathrm{mmol} / \mathrm{L}$; range 1.3 - $4.45 \mathrm{mmol} / \mathrm{L})$ and multi-organ failure (OF $\geq 2 ; n=34)$ (3.70 (0.81) $\mathrm{mmol} / \mathrm{L}$; range $1.4-4.6 \mathrm{mmol} / \mathrm{L}$ ).

The lactic acid levels were also found to be associated with positive cultures. The lactic acid levels $(3.76(0.97) \mathrm{mmol} / \mathrm{L})$ in patients with any type of positive culture $(n=35)$ were significantly higher than in those with negative cultures (2.95 (0.98); $p=0.0001$ ). At the critical threshold of $4 \mathrm{mmol} / \mathrm{L}$, there were significant differences between culture-negative and culture-positive cohorts (Table 1). The lactic acid levels $\geq 4 \mathrm{mmol} / \mathrm{L}$ had a positive predictive value of $70.4 \%$ and negative predictive value of $78.08 \%$ with a specificity of $87.7 \%$ for a positive bacterial culture in PAS patients.

\section{Discussion}

A product of anaerobic metabolism related to hypovolaemia and hypoxia, serum lactic acid levels is a known parameter for the early identification of sepsis in general. ${ }^{[1]}$ A review of 15 studies using lactic acid as a sepsis marker in adult populations suggested a higher specificity compared with sensitivity in indicating sepsisrelated prognosis and mortality. ${ }^{[1]}$ Lactic acid was an indicator of poor perfusion, and higher levels of lactate were consistent with a more compromised circulatory system. The above review identified lactic acid levels $>4 \mathrm{mmol} / \mathrm{L}$ as necessitating critical care in sepsis; however, there were limitations of evidence levels and biases in various studies included in the review. ${ }^{[1]}$

Table 1. Lactic acid levels in PAS subjects: Association with severity and culture results.

\begin{tabular}{|c|c|c|c|}
\hline \multirow[b]{2}{*}{$\begin{array}{l}\text { Lactic acid } \\
(\mathrm{mmol} / \mathrm{L})\end{array}$} & \multicolumn{2}{|c|}{ Severity of PAS } & \multirow[b]{2}{*}{$p$-value ${ }^{*}$} \\
\hline & $\begin{array}{l}\text { Non-severe sepsis } \\
(N=42), n(\%)\end{array}$ & $\begin{array}{l}\text { Severe sepsis } \\
(N=58), n(\%)\end{array}$ & \\
\hline$<4$ & $37(50.7)$ & $36(49.3)$ & 0.000 \\
\hline \multirow[t]{3}{*}{$\geq 4$} & $5(18.5)$ & $22(81)$ & \\
\hline & \multicolumn{2}{|c|}{ Culture results } & \\
\hline & $\begin{array}{l}\text { Negative (N=65), } \\
n(\%)\end{array}$ & $\begin{array}{l}\begin{array}{l}\text { Positive } \\
(N=35), n(\%)\end{array} \\
\end{array}$ & \\
\hline$<4$ & $57(87.7)$ & $16(45.7)$ & 0.000 \\
\hline$\geq 4$ & $8(12.3)$ & $19(54.3)$ & \\
\hline
\end{tabular}

The evidence for the role of lactic acid as a potential marker for critical care in PAS is still scanty. ${ }^{[2-5]}$ In pregnant patients, lactic acid levels $>2 \mathrm{mmol} / \mathrm{L}$ indicate tissue hypoxia and $>4 \mathrm{mmol} / \mathrm{L}$ is associated with severe tissue hypoxia. ${ }^{[4]}$ It is also considered to be one of the reasons for metabolic acidosis. ${ }^{[4]}$ Notably, a study of 159 pregnant women showed that increased lactic acid levels were associated with adverse maternal and perinatal outcomes, such as the need for critical care, positive blood cultures, preterm delivery and prolonged hospital admission. ${ }^{[2]}$ The mean lactic concentration was higher in patients admitted to the intensive care unit $(2.6 \mathrm{v}$. $1.6 \mathrm{mmol} / \mathrm{L} ; p=0.04)$ and in those with positive blood cultures $(2.2 \mathrm{v} .1 .6 ; p<0.01) .^{[2]}$

With growing evidence in support of the importance of evaluating lactic acid levels when making clinical decisions, a popular scoring system for identification of sepsis in pregnant and postpartum women, the SOS, now considers lactic acid measurement as a standard practice when evaluating the possibly septic obstetric patient. ${ }^{[3]}$ Lactic acid levels form a key part of the Surviving Sepsis Campaign Care bundle and are used as a guide to determine the degree of organ hypoxia, as well as whether organ perfusion improves with volume replacement. ${ }^{[4]}$

\section{Study limitations and strengths}

The main limitation of our study was the lack of a control group without sepsis. We could not perform serial lactic acid estimations to establish lactic acid as a monitoring tool in PAS for logistic and financial reasons. The lactic acid therefore formed part of the initial care bundle of the patients. Study strengths included it being the first of its kind to investigate lactic acid levels in an obstetric population in a low-income setting. Another strength was that we employed a robust sample size. The importance of the study is further indicated by looking at the high burden of maternal sepsisassociated morbidity and mortality in low-income countries, and the urgent need to develop cheaper diagnostic tools with wide availability.

\section{Conclusion}

Our study demonstrated reasonable specificity of lactic acid level $\geq 4 \mathrm{mmol} / \mathrm{L}$ for severe PAS in our population. A serum lactic acid level $>3 \mathrm{mmol} / \mathrm{L}$ at admission had considerable association with the occurrence of organ failure. Thus, lactic acid estimations can be used as a useful guide for triaging PAS patients for severity and timely critical care. Further, our prospective study revealed that the lactic acid level of $\geq 4 \mathrm{mmol} / \mathrm{L}$ had significant statistical association with positive cultures. We strongly believe that if lactic acid levels are elevated, microbial identification and prompt antibiotic administration should be considered.

Overall, our study highlights the fact that lactic acid levels have a role to play in detecting the severity of PAS, similar to that played with non-pregnant adults with PAS. There is a strong need to develop multicentre case control evidence for better quantification of the role of lactic acid in PAS.

\author{
Acknowledgements. None. \\ Author contributions. All authors contributed equally to the preparation of the \\ manuscript. \\ Funding. None.
}

Conflicts of interest. None. 


\section{RESEARCH}

1. National Guideline Centre. Sepsis: Recognition, Assessment and Early Management. London National Institute for Health and Care Excellence, 2016. https://www.ncbi.nlm.nih.gov/pubmedhealth/ PMH0088862/ (accessed 14 May 2017).

2. Albright CM, Ali TN, Lopes V, Rouse DI, Anderson BL. Lactic acid measurement to identify risk of morbidity from sepsis in pregnancy. Am J Perinatol 2015;32(5):481-486. https://doi. org/10.1055/s-0034-1395477

3. Albright CM, Mehta ND, Rouse DJ, Hughes BL. Sepsis in pregnancy: Identification and management. J Perinat Neonatal Nurs 2016;30(2):95-105. https://doi.org/10.1097/JPN.0000000000000159

4. Brown KN, Arafeh JM. Obstetric sepsis: Focus on the 3-hour bundle. J Perinat Neonatal Nurs 2015;29(3):213-221. https://doi.org/10.1097/JPN.0000000000000115

5. Galvão A, Braga AC, Gonçalves DR, Guimarães JM, Braga J. Sepsis during pregnancy or the postpartum

period. J Obstet Gynaecol 2016;36(6):735-743. https://doi.org/10.3109/01443615.2016.1148679
6. Rhodes A, Evans LE, Alhazzani W, et al. Surviving Sepsis Campaign. International guidelines for management of sepsis and septic shock: 2016. Intensive Care Med 2017;43(3):304-377. https://doi. org/10.1007/s00134-017-4683-6

7. Oud L. Pregnancy associated severe sepsis. Curr Opin Obstet Gynecol 2016;28(2):73-78. https://doi. org $/ 10.1097 / G C O .0000000000000250$

Accepted 16 February 2018. 